\title{
Interferometric Surface Relief Measurements with Subnano/Picometer Height Resolution
}

\author{
Evgeny Sysoev $^{1}$, Sergey Kosolobov ${ }^{2}$, Rodion Kulikov ${ }^{1}$, Alexander Latyshev ${ }^{2}$, Sergey Sitnikov², Ignat \\ Vykhristyuk $^{1}$ \\ ${ }^{1}$ Technological Design Institute of Scientific Instrument Engineering, Siberian Branch of the Russian Academy of Sciences \\ (TDI SIE SB RAS), 41, Russkaya str., 630058, Novosibirsk, Russia, evsml@mail.ru \\ 2 A.V. Rzhanov Institute of Semiconductor Physics, Siberian Branch of the Russian Academy of Sciences (ISP SB RAS), 13, \\ Academika Lavrentieva Prospekt,630090,Novosibirsk,Russia,kosolobov@isp.nsc.ru
}

\begin{abstract}
We present an optical interference system nanoprofiler MNP-1 designed for high-precision noncontact measurement of surface relief with subnanometer resolution (root mean square of measured values), based on partial scanning of interference signal. The paper describes the construction of the measurement system with Linnik interferometer and the algorithm for nanorelief surface reconstruction. Experimental measurement results of silicon sample with profile height of surface structure of one interatomic distance obtained by MNP-1 are shown. It was proposed to use an atomically smooth surface as the reference mirror in the interferometer MNP-1 that allowed us to measure monatomic steps of the presented silicon sample. Monatomic steps of $0.31 \mathrm{~nm}$ in height on silicon (111) surface were measured with resolution up to $5 \mathrm{pm}$.
\end{abstract}

Keywords: nanorelief, monatomic steps, low-coherence, white light interferometer, subnanometer resolution.

\section{INTRODUCTION}

Providing high resolution, when measuring the height of the surface relief, is an actual problem both in scientific research [1] and industrial production [2]. It is well known that the optical interference measuring techniques, especially white light interferometry, solve this problem, since the phase of the interference signal is very sensitive to small changes in the optical path difference between the interfering waves.

For precise measuring of surface nanorelief the wellknown methods of phase shifting interferometry (PSI) are used. These methods are based on the phase calculation for the light scattered in the interferometer arms [3]. Typically, PSI measurement systems use Michelson, Linnik or Mirau interferometers [4], [5]. In this case for surface nanorelief measurement a scanning of interference signal phase is performed by changing the optical path difference of light between reference and measurement interferometer arms [6]. During the scanning process, a set of interferograms is recorded. The calculation of surface nanorelief height of measured sample is carried out by means of these interferograms at a known wavelength of used light source.

The recorded interferograms are the result of the interaction of two wavefronts formed by 'etalon', which is used as an interferometer reference surface and the surface of the measurement object. At the surface nanorelief calculation both surfaces 'etalon' and the measurement object influence the measurement results. The analysis of measurement results shows that measurement error is defined first of all by the quality of interferometer reference surface. When roughness of reference interferometer surface exceeds a height of surface relief of measurement sample a big error in the measurement results is observed.

As a rule, the interference systems designed for the measurement of surface nanorelief use flat optical mirrors made by spraying of $\mathrm{Al}$ on high quality glass substrate as an interferometer reference surface. The roughness of such mirrors is about $2 \mathrm{~nm}$. To provide subnanometer height resolution the reference mirrors must have lesser roughness.

There is the approach to decrease the influence of reference mirror roughness. In the work [7] the authors proposed to carry out some uncorrelated measurements of supersmooth mirror and then to average the measurement results to get reference surface. Further, this reference surface is "subtracted" from subsequent measurements. This technique is similar to interferometer calibration by measuring the caliber surface. The surface relief of caliber is preliminary measured by a metrologically certified system. The data received in the process of interferometer calibration is also used to correct the measurement results.

Significant progress was achieved using the molecular beam epitaxy (MBE) technology for the formation of flat 
smooth solid-state surfaces [8]. In this case there is a real opportunity to create the atomically smooth surface. By authors' opinion to decrease the influence of reference mirror roughness the most perspective is to use the atomically smooth surface as reference mirror of interferometer. The application of such reference mirror under the method of partial correlogram scanning allows us to decrease the error of surface nanorelief measurement and significantly improve resolution capability by height up to 5 picometers.

The paper describes the method of partial correlogram scanning assigned for measurement of 3D surface nanorelief and the nanoprofiler MNP-1. Experimental measurement results of silicon sample with height of surface structure of one monoatomic layer obtained by nanoprofiler MNP-1 are presented here. The results of using atomically smooth surface as the interferometer reference mirror in the measurement system MNP-1 to obtain the picometer resolution also are shown in the paper.

\section{THE PARTIAL CORRELOGRAM SCANNING METHOD FOR SURFACE NANORELEIF MEASUREMENT}

The principle of surface nanorelief measurement is based on the method of partial correlogram scanning and described in detail in [9]. According to this method the correlograms are registered for different parts of measurement surface as shown in Fig.1. The correlogram is the function of interference intensity depending on the phase changing. Height difference $\Delta h$ between different surface parts leads to that the recorded correlograms have different phase shift $\Delta \Phi$ (Fig.1.). They are related by the following expression:

$$
\Delta h=\frac{\Delta \Phi}{2 \pi} \cdot \frac{\bar{\lambda}}{2}
$$

where $\bar{\lambda}$ is the effective wavelength.

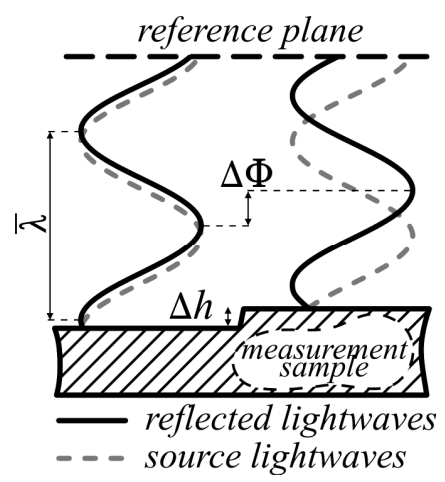

Fig.1. Measurement method: different phase shift of lightwaves reflected from the measurement sample.

At the measurement of surface nanorelief by this method only a part of correlogram (which includes 2-3 periods) is registered in each point. Correlograms are obtained in the process of changing the optical path difference between interfering lightwaves. The scanning process of phase is carried out with selected scanning step, for example, by piezoelectric transducer. Interferograms which are formed for each scanning step can be registered by multielement photodetector and then digitized and transmitted to personal computer. After the scanning process for each points of measurement area (these points correspond to photodetector elements) the correlograms are formed. For example, two correlograms which correspond to two points on the surface with different heights are shown in Fig. 2.

Phase difference $\Delta \Phi$ between obtained correlograms is calculated from the following expression:

$$
\Delta \Phi=\frac{\Delta n}{2 N} \cdot 2 \pi
$$

where $2 N$ is the amount of scanning steps necessary for changing the optical path difference between interfering beams at half of effective wavelength, and $\Delta n$ is the shift between correlograms (calculated in the number of scanning steps).

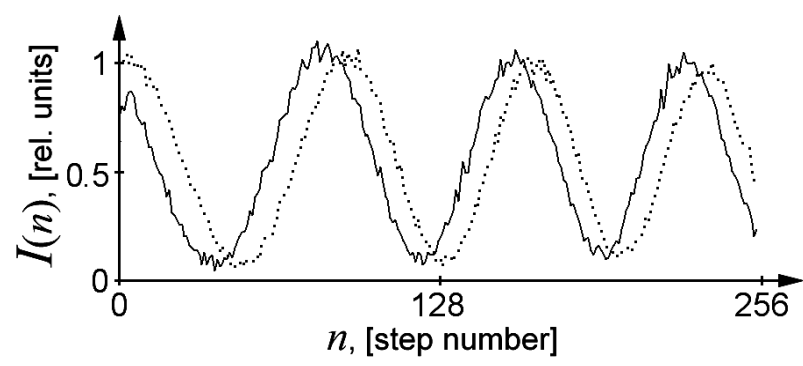

Fig.2. The correlograms for two different points of the surface: $I(n)$ is the light intensity, $n$ is the number of scanning step.

The $N$ and $\Delta n$ values are calculated by the displacement of the first correlogram $I_{1}(n)$ relative to the second one $I_{2}(n)$. Displacement when the value of standard deviation reaches a minimum corresponds to the desired value of shift:

$$
\sigma(\Delta n)=\sqrt{\frac{2}{K} \cdot \sum_{n=K / 4}^{3 K / 4-1}\left[I_{1}(n)-I_{2}(n+\Delta n)\right]^{2}} \underset{\Delta n \in[-K / 4, K / 4]}{\rightarrow} \min
$$

where $K=256$ is the number of registered interferograms.

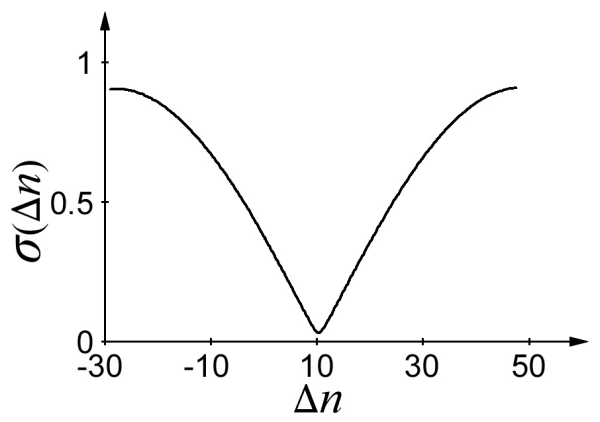

Fig.3. The calculation of shift between two correlograms: $\sigma(\Delta n)$ is the standard deviation, $\Delta n$ is the shift between two correlograms. 
At the definition of $N$ value the second correlogram is a copy of the first one but inverted in intensity. In the case of definition of $\Delta n$ value the two correlograms corresponding to different part of measured surface are used. The example of the dependence of standard deviation $\sigma$ on assigned displacement at definition of $\Delta n$ value is shown in Fig.3.

The calculation of phase difference between all correlograms reconstructs the surface nanorelief.

A distinctive feature of the method of partial correlogram scanning is that the required scanning range is less than $1 \mu \mathrm{m}$ and the scanning involves displacement of reference mirror only. This allows one to significantly increase the measurement speed and simplify the design of the microscope. Moreover, an absolute phase value is not used under the phase difference calculation. In the case of the absolute phase measurements the relief height is unambiguously defined in the phase variation range of $2 \pi$. The calculation of relief height by the method of partial correlogram scanning is based on calculation of phase variations. A priori, if we assume that the height difference between two neighbor parts of measured surface does not exceed $\bar{\lambda} / 4$, then this method allows measuring the surface nanorelief height in the range limited by coherence length of the used light source. At that the ambiguity in the measurement of surface nanorelief arises only at a local height difference of $\pm \bar{\lambda} / 4$. That feature allows avoiding the ambiguity of relief height calculation.

\section{MNP-1 NANOPROFILER}

Morphology of sample surface was analyzed by the method of partial correlogram scanning by means of nanoprofiler MNP-1 [10]. This measurement system was developed and produced at TDI SIE SB RAS. Nanoprofiler MNP-1 shown in Fig.4. consists of optomechanical module 1 , electronic module 2, and computer 3 with specially designed software.

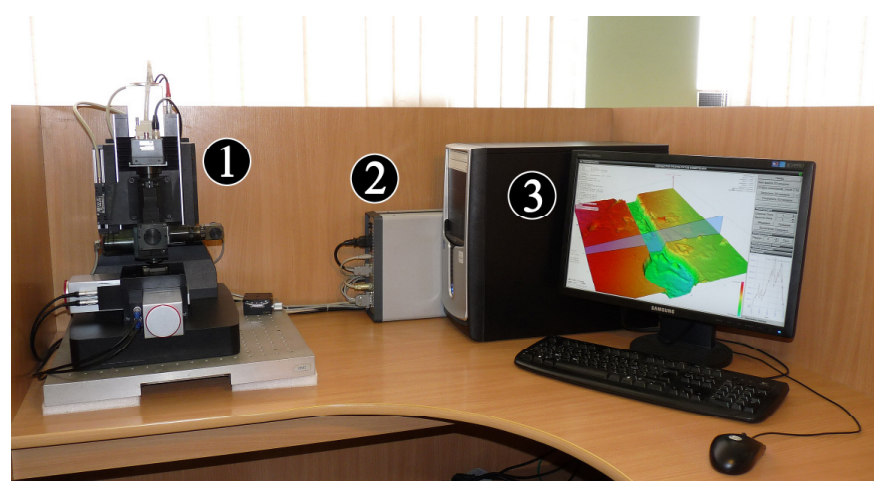

Fig.4. Nanoprofiler MNP-1: 1 - optomechanical module, 2 - electronic module, 3 - computer.

Detailed scheme of nanoprofiler MNP-1 is shown in Fig.5. Optomechanical module includes the base with horizontal $X Y$-axes stage 5 and vertical $Z$-axis stage 4 fixed on holder. Stages controller installed inside the computer 3 operates the carriage position of XY-axes and Z-axis stages. Main part of the interferometer 1 is mounted on the carriage of $Z$-axis stage 4 . The carriage position of $Z$-axis stage is controlled by linear displacement sensor 14 connected to the electronic module 2. The computer 3 controls the light source 6 , the piezoelectric transducer 9 and the power supply of CCD camera 15 by electronic module 2 via USB 2.0 interface. The CCD camera 15 registers images and through the frame grabber transmits them to the computer. To carry out the measurements the investigated object 13 is placed on $X Y$ axes stage 5 .

The interferometer was designed according to the scheme of Linnik micro-interferometer [11]. The light wave from a source of partially coherent light 6 (used LED) passes through a collimating objective 10 and gets onto the beam splitter cube 7, where it is divided by the amplitude into two parts. One part of light wave propagates in the reference arm, another one - in the measurement arm of interferometer. In the reference arm of the interferometer the light wave passes through the microobjective 12 and gets onto the surface of reference mirror 8 fixed on the piezoelectric transducer 9. Propagating in the measurement arm of the interferometer the light wave passes through the microobjective 11 and gets onto the surface of measurement object 13 . The light partially scattered and reflected by the surfaces of reference mirror and measurement object is registered by matrix of the $\mathrm{CCD}$ camera 15 . The interference phenomena are observed under the condition that the optical path difference of light between reference and measurement arms does not exceed the coherence length of the used light source.

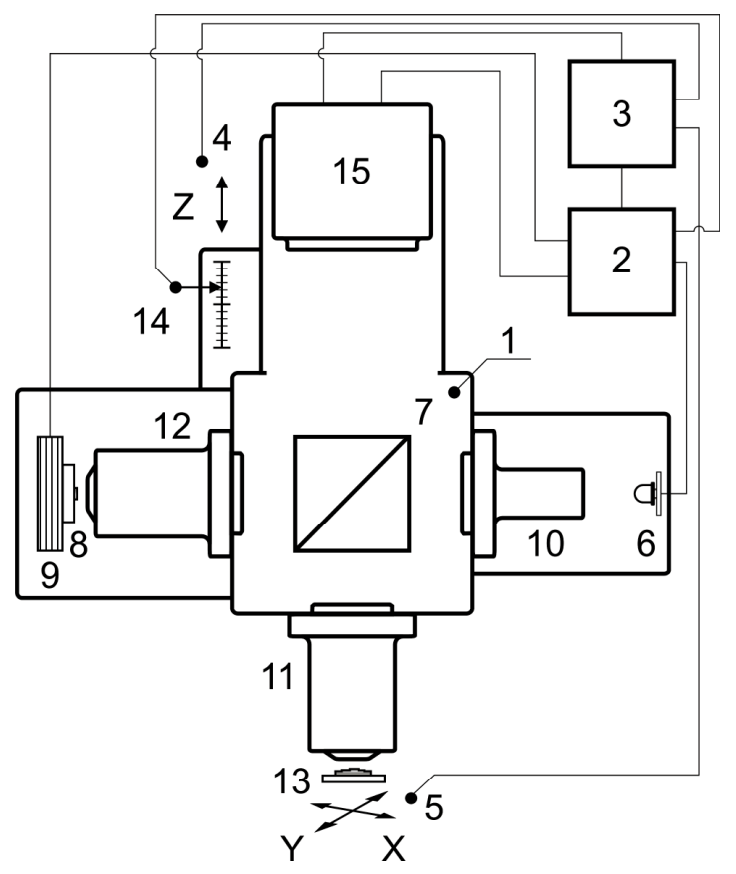

Fig.5. Scheme of nanoprofiler MNP-1: 1-main part of interferometer, 2 -electronic module, $3-\mathrm{PC}, 4-Z$-axis stage, $5-X Y$-axes stage, 6 - low coherence light source,

7 - beam splitter, 8 - reference mirror, 9 - piezoelectric transducer, 10 - collimating objective, 11-12 -micro objectives,

13 - measuring surface, 14 - linear displacement sensor,

15 - CCD-camera. 


\section{EXPERIMENTAL RESULTS OBTAINED BY MNP}

In order to determine the height resolution of the interferometric system nanoprofiler MNP-1 a special surface of silicon sample was used. This measurement sample was designed at A.V. Rzhanov Institute of Semiconductor Physics, Siberian Branch of the Russian Academy of Sciences (ISP SB RAS). The investigated sample was prepared by thermal annealing of the silicon (111) crystal under ultrahigh vacuum conditions. After thermal annealing, the samples were evacuated from UHV (ultra-high vacuum) chamber and their morphology was analyzed at ambient conditions. Current sample of silicon crystal contains on the surface the structure of monoatomic steps with height in one atomic spacing of crystal lattice of silicon. The step height was measured by the producer using AFM (Solver P47H, Integra Aura, NT MDT) and was equal to $3.14 \AA$. The standard silicon cantilevers were used for profile measurements both in contact and in semi-contact modes at ambient condition. Fig.6. represents the measurement result, namely: the AFM-image (semi-contact mode) of the silicon surface on the area $80 \times 80 \mu \mathrm{m}^{2}$ with the atomic steps $0.31 \mathrm{~nm}$ in height, bounded two-dimensional "negative island".

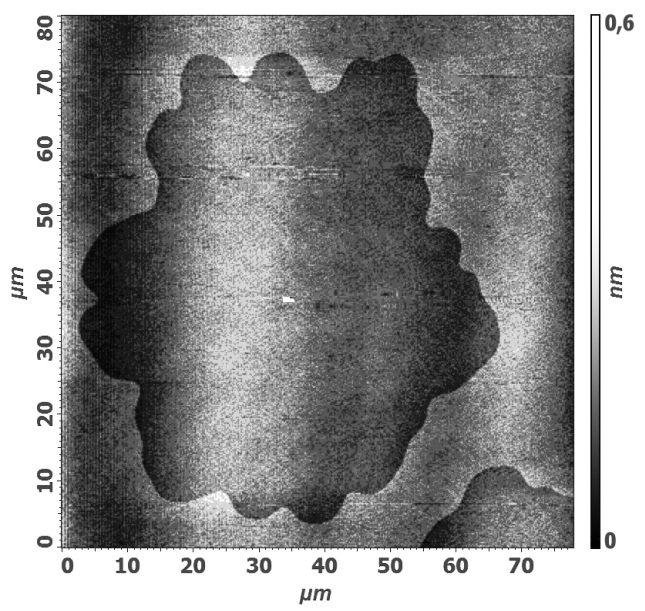

Fig.6. AFM-image $80 \times 80 \mu \mathrm{m}^{2}$ of silicon surface with negative island of monoatomic depth.

The measurements of proposed sample made by the nanoprofiler MNP-1 were carried out at the parameters of the measurement system which are given further. The microobjectives with magnification $20^{\times}$and numerical aperture $(N A) \quad 0.4$ were used in the reference and measurement arms of Linnik interferometer. As a light source in the interferometer we have applied the calibrated light source with effective wavelength $630 \pm 0.5 \mathrm{~nm}$. The calibration of light source was carried out by the spectrometer LINOS QWave with resolution ability of $0.5 \mathrm{~nm}$. A preliminary calibration of the spectrometer was carried out by He-Ne laser with wavelength $632.9918 \mathrm{~nm}$ stabilized by Lamb dip with relative non-stability of optical frequency not more than $10^{-8}$. Also, the value of wavelength can be changed by the optical scheme of interferometer. In work [12] was shown that the value of wavelength depends on NA. Estimated value of the wavelengths difference for used microobjectives $20^{\times}$with NA $=0.4$ and $\lambda=630 \mathrm{~nm}$ is less than $4 \%$. In case of measurement of monoatomic steps with height $3.14 \AA$ it leads to insignificant systematic error.

CCD camera Hitachi KP-F120CL was used for interferograms registration. The camera pixel size is $6.45 \mu \mathrm{m} \times 6.45 \mu \mathrm{m}$. The pixel size in the measurement area is equal to $0.32 \mu \mathrm{m} \times 0.32 \mu \mathrm{m}$. The registration process of interferograms was performed by averaging of four frames.

Fig.7. shows results of the first set of measurements. They were performed by the nanoprofiler MNP-1. In the reference arm of MNP-1 interferometer an optical flat mirror made by vacuum spraying of $\mathrm{Al}$ on high quality glass substrate was used. Three-dimensional surface relief reconstruction (Fig.7.a)) and corresponding plot of height distribution (histogram) for the sample region (Fig.7.b), Fig.7.c)) clearly show that resolution of this method is good enough for the profile measurements of the surface with average roughness (half width at half maximum) of the order of $1 \mathrm{~nm}$. Note here that we consider height resolution as a rms (root mean square) of measured values of height.

Unfortunately, instrumental function of interferometer introduces phase distortions which are not allowed resolving the isolated atomic steps which are situated on the analyzed surface.

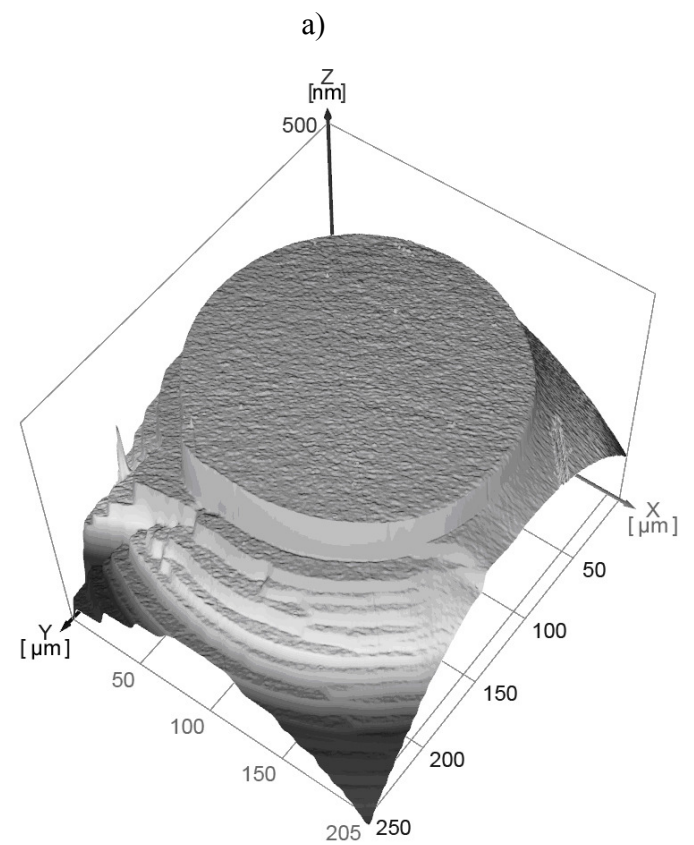

b)

c)

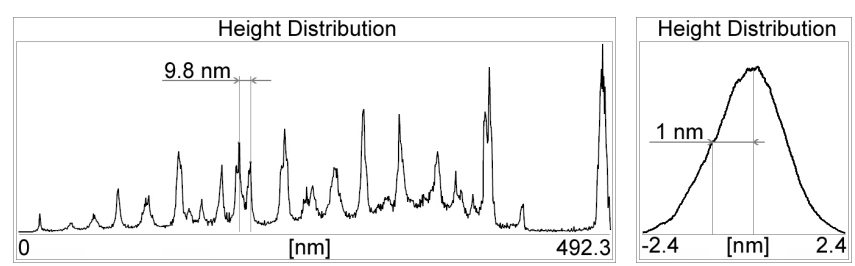

Fig.7. The reconstructed 3D model of surface a) and height distribution for the whole area $b$ ) and central region c). 
To eliminate the influence of instrumental function the differential measurement of surface nanorelief was proposed. Two measurements of the same sample situated in the different positions in the plane $X Y$ were carried out: first is the initial position, the second is a position shifted by about $15 \mu \mathrm{m}$. Further, the measurement results which were obtained for two positions of sample were subtracted one from another. The result of differential measurements of surface nanorelief containing monoatomic steps is shown in Fig.8. Thus, the differential measurement of surface nanorelief performed by nanoprofiler MNP-1 allowed us to register the structure of monoatomic steps on the sample surface.

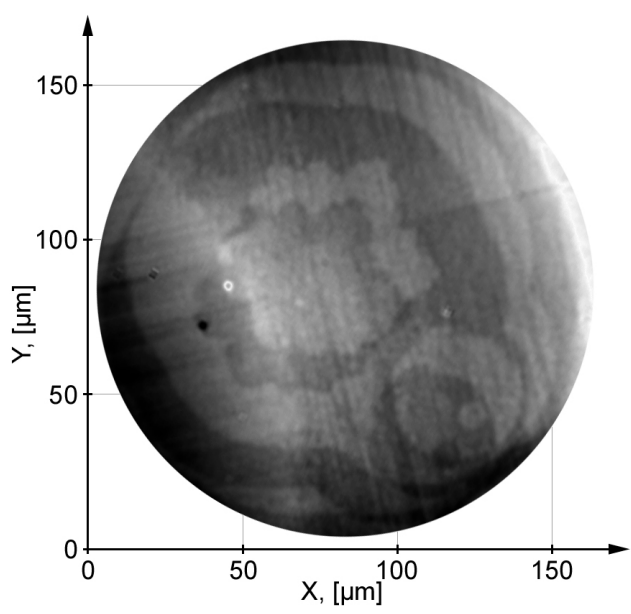

Fig.8. Results of differential measurements of surface nanorelief with monoatomic steps.

\section{PICOMETER HEIGHT PROFILOMETRY BY USING ATOMICALLY SMOOTH MIRROR}

In order to increase the height resolution of nanoprofiler MNP-1, special silicon sample containing wide and extremely smooth surface area was designed by ISP SB RAS. We proposed to place this surface in the reference arm of the interferometer and use it as a reference mirror.

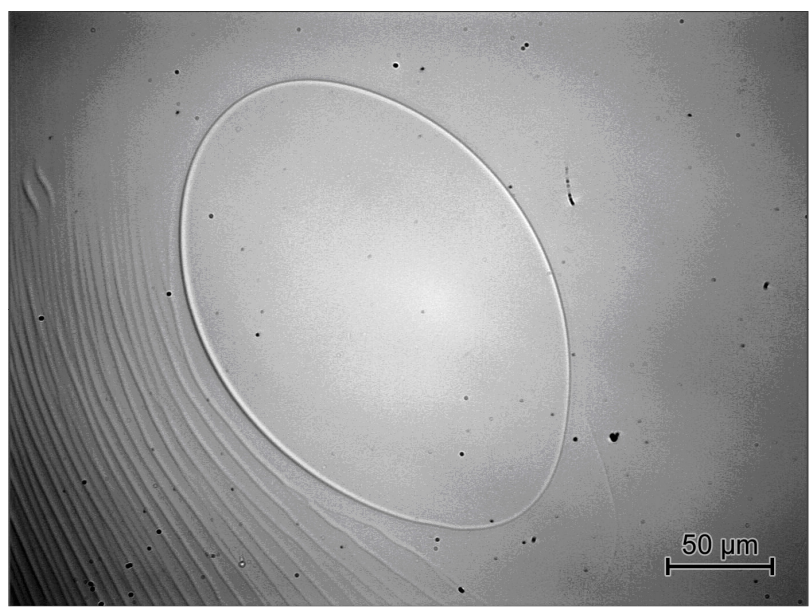

Fig.9. Optical image of the reference mirror with atomicallysmooth region of silicon.
This sample contains the surface region (atomicallysmooth singular terrace) with an average roughness $R_{a}$ below $0.03 \mathrm{~nm} \mathrm{[8].} \mathrm{Microphotograph} \mathrm{of} \mathrm{this} \mathrm{reference}$ mirror obtained in MNP-1 is shown in Fig.9. Atomically smooth region of the surface in the form of ellipse with sizes $200 \mu \mathrm{m} \times 160 \mu \mathrm{m}$ is clearly seen at the center of image.

After installation of atomically smooth surface in the reference interferometer arm, the measurement of silicon sample with relief structure height of one interatomic distance was performed again.

a)

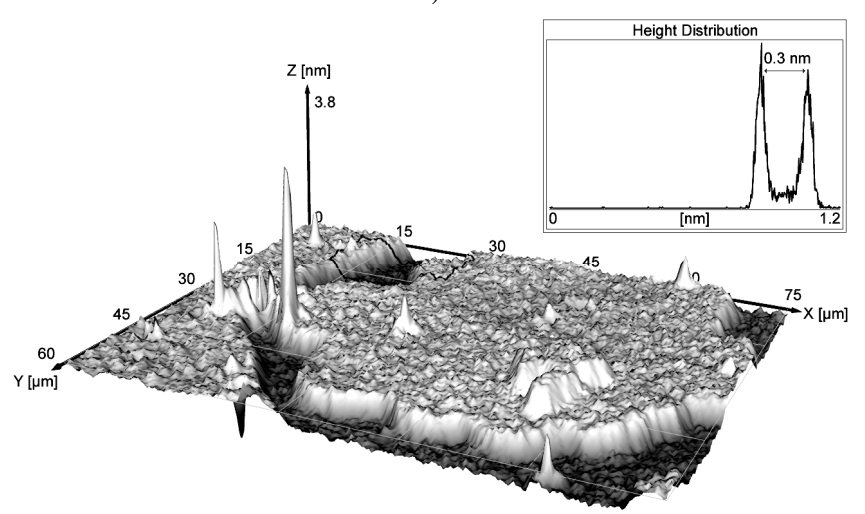

b)

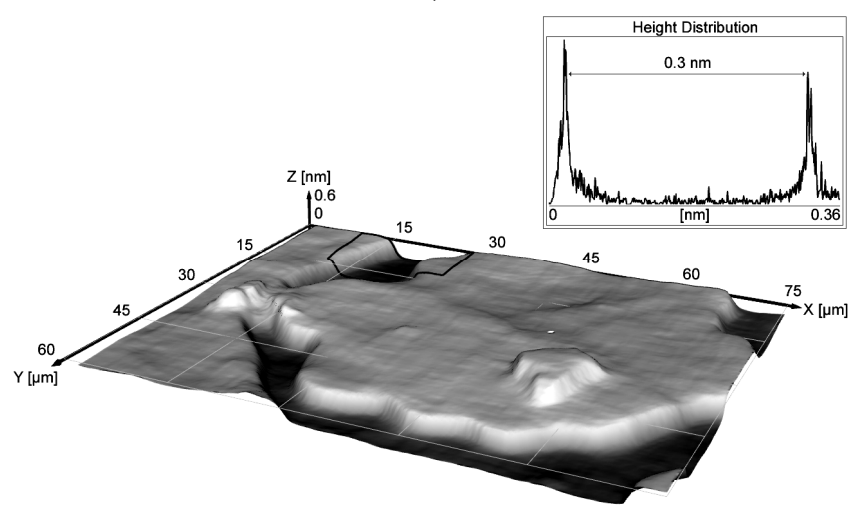

Fig.10. 3D surface relief reconstruction of Si (111) sample without a) and after spatial filtering b) with plot of height distributions for selected areas (insets).

Fig.10. represents the measurement results of the same sample surface area as in Fig.6., Fig.7. (central region), and Fig.8. which were obtained by the new reference mirror. The measurement results of the sample without filtering are shown in Fig.10.a).

In the case of preliminary knowledge of object morphology, the application of various filters for resolution increase will be effective. In particular, under the measurements of monoatomic steps one can use averaging and(or) median filtering with window width about half of characteristic size of steps. The results of measurements of monoatomic step with size about $20 \mu \mathrm{m}$ are shown in Fig.10.a). In this case the filter with size of $10 \mu \mathrm{m}$ was used (Fig.10.b)). Thus, the application of such filter is correct and 
allows us to fulfill the measurement of monoatomic step with higher accuracy.

It was shown that even without filtration of measured surface relief the use of atomic smooth mirror allows us to drastically increase the height resolution and measure the height of monoatomic steps.

The measurements of step height and measurements ambiguity were carried out by standard method using the histogram of height distribution. Height of steps (Fig.10.a), Fig.10.b)) was calculated as a distance between graph extrema, and the ambiguity of height definition of steps was estimated by standard deviation. The height distributions shown at insets of Fig.10.a), Fig.10.b) allow us to estimate height resolution of this method as 30 and 5 pm for case without filtering and after applying spatial filter, respectively.

\section{CONCLUSION}

The experimental results carried out by MNP-1 at the surface relief measurement of silicon sample with monoatomic layers structure are shown. Due to differential measurements, the nanoprofiler MNP-1 has been able to detect monoatomic structure.

Technical parameters of MNP-1 were following: Objectives $20^{\times}$; frame rate $30 \mathrm{fps}$;

CCD-camera matrix $1392 \times 1040$ pixels; pixel size $6.45 \mu \mathrm{m}$; $\mathrm{s} / \mathrm{n}$ ratio $60 \mathrm{~dB}$;

Measurement range up to $20 \mu \mathrm{m}$;

Height resolution not worse than $0.1 \mathrm{~nm}$;

Lateral resolution $\quad 0.79 \mu \mathrm{m}$ (for $630 \mathrm{~nm}$ );

Measurement area $\quad 0.45 \mathrm{~mm} \times 0.33 \mathrm{~mm}$;

Measurement speed $20 \mu \mathrm{m}$ to 10 seconds.

For improvement height resolution of MNP-1, special silicon sample (designed at ISP SB RAS) containing wide and extremely smooth surface area (atomically smooth mirror) was proposed to use as a reference mirror in the interferometer.

The obtained results show that using an atomically smooth surface as the reference mirror of the interferometer and the method of partial correlogram scanning drastically improve the height resolution of nanoprofiler below $30 \mathrm{pm}$ and allow one to visualize monatomic steps on silicon surface.

It is essential that preliminary knowledge of measurement object structure allowed us to use the processing of the measurement result and to obtain resolution by height 5 picometers.

As an extension of the work it is proposed to carry out experiments to measure surface relief of sample with monoatomic structures by means of interferometer which contains both atomic smooth reference mirror and stable laser radiation source.

\section{REFERENCES}

[1] Guilemany, J.M., Miguel, J.M., Armada, S., Vizcaino, S., Climent, F. (2001). Use of scanning white light interferometry in the characterization of wear mechanisms in thermal-sprayed coatings. Materials Characterization, 47 (3-4), 307-314.

[2] De Groot, P., Biegen, J., Clark, J., Lega, X.C., Grigg, D. (2002). Optical interferometry for measurement of the geometric dimensions of industrial parts. Applied Optics, 41 (19), 3853-3860.

[3] De Groot, P. (2011). Coherence scanning interferometry. In Optical Measurement of Surface Topography. Springer, 187-208.

[4] Niehues, J., Lehmann, P., Xie, W. (2012). Low coherent Linnik interferometer optimized for use in nano-measuring machines. Measurement Science and Technology, 23 (12), 125002.

[5] Windecker, R., Fleischer, M., Körner, K., Tiziani, H.J. (2001). Testing micro devices with fringe projection and white-light interferometry. Optics and Lasers in Engineering, 36 (2), 141-154.

[6] Hariharan, P., Malakara, D. (1995). Selected Papers on Interference, Interferometry, and Interferometric Metrology. SPIE Optical Engineering Press.

[7] Creath, K., Wyant, J.C. (1990). Absolute measurement of surface roughness. Applied Optics, 29 (26), 38233827.

[8] Sitnikov, S.V., Kosolobov, S.S., Shcheglov, D.V., Latyshev, A.V. (2011). Method of forming flat smooth surface of solid material. Patent RU 2453874.

[9] Sysoev, E.V. (2007). White-light interferometer with partial correlogram scanning. Optoelectronics, Instrumentation and Data Processing, 43 (1), 83-89.

[10] Sysoev, E.V., Vykhristuk, I.A., Kulikov, R.V., Potashnikov, A.K., Razum, V.A., Stepnov, L.M. (2010). Interference microscope-profilometer. Optoelectronics, Instrumentation and Data Processing, 46 (2), 198-205.

[11] Rosenberg, G.V. (1953). Interferencionnaya mikroskopiya. Uspehi fizicheskih nauk, L (2), 279281. (in Russian)

[12] Dubois, A., Selb, J., Vabre, L., Boccara, A.-C. (2000). Phase measurement with wide-apperture interferometers. Applied Optics, 39 (14), 2326-2331.

Received March 03, 2017. Accepted September 18, 2017. 